\title{
Precoding and Feedback Schemes for a MIMO Backhaul Link in the Presence of Interference
}

\author{
Daniel Sacristán-Murga* and Antonio Pascual-Iserte* ${ }^{* \dagger}$ \\ ${ }^{*}$ Centre Tecnològic de Telecomunicacions de Catalunya (CTTC), Spain \\ ${ }^{\dagger}$ Dept. of Signal Theory and Communications - Universitat Politècnica de Catalunya (UPC), Spain \\ Email: daniel.sacristan@cttc.es, antonio.pascual@upc.edu
}

\begin{abstract}
The focus of this paper is on the precoding and feedback strategies for a radio backhaul link between an access base station (ABS) and the hub base station (HBS) in the presence of mobile stations (MSs). In this paper it is assumed that the access and the backhaul links are simultaneous and use the same frequency band. Two feedback links are exploited at the ABS in order to optimize the communication through the radio-backhaul link: the MSs feed back the channel state information (CSI) of the channels between the ABS and the MSs, and the HBS feeds back the statistics of the received interference. Using this information, a transmission strategy is proposed for the ABS in order to transmit to the HBS without interfering to the MSs and at the same time minimizing the effect of the interference received at the HBS.
\end{abstract}

\section{INTRODUCTION}

During the last years there has been considerable research in the field of multiple antenna communications over the wireless channel, and it has been shown to provide large gains in channel capacity [1] and resilience to fading [2]. In order to fully achieve this performance improvement, complete knowledge of the channel state information (CSI) is required at the transmitter [3]. In systems where channel reciprocity does not hold, a feedback link can be used to send the CSI estimated at the receiver to the transmitter. Extensive work has been done on the design of feedback strategies, with focus on the cases of point-to-point multi-input-multi-output (MIMO), broadcast channel (BC), and to a lesser extent to the interference channel.

This paper presents a novel scenario, which emerges from the planing of interference-aware all-wireless networks that feature aggressive frequency reuse. We consider a network topology composed of access and backhaul links which are both wireless. Having a wireless backhaul link greatly reduces the cost in time and resources of the deployment of the network. In the access network, multiple access base stations (ABSs), which are fixed in space, communicate over the wireless channel with different mobile stations (MSs) which have a given mobility. In the backhaul links the hub base stations (HBSs), which are connected directly to the core

The research leading to these results has received funding from the European Community's Seventh Framework Programme (FP7/2007-2013) under grant agreements no. 248267 (BuNGee) and no. 216715 (NEWCOM++). It has received further support from the Catalan Government under grant 2009 SGR 891, and by the Spanish Government under project TEC2008-06327-C03 (MULTI-ADAPTIVE). operator's network, communicate with the ABSs (each HBS handles the aggregated traffic of multiple ABSs). Traditional communication networks feature a wired backhaul link, which translates into large deployment times and costs. Recently there is a trend to evolve the backhaul link to be wireless, as for example in the WiMAX standard [4], [5]. The novelty of the scenario presented in this work lies in the fact that an array of omnidirectional antennas is considered at both ends of the radio backhaul link, i.e., the links can be adapted dynamically to mitigate incoming interference. This also removes the planning needed when using very directive antennas in the wireless backhaul link. Besides, in order to increase the spectral efficiency of the system, the same frequencies are used for the backhaul and the access networks, which means that cross-segment (or cross-system) interference has to be taken care of. This is also a novel deployment.

This work considers a cross-segment (or cross-system) design of the transmit precoding matrix of the ABS for the communication with the HBS under the following design considerations: first, the backhaul link should be completely transparent to the access network, i.e., the signals sent to the HBS should not cause interference to the MSs, and second, the design should also minimize the effect of interference at the HBS. The interference at the HBS can be due to external sources and, if the system is full-duplex, also to the signals sent by the MSs.

In order to satisfy these design criteria, two limited feedback links are proposed to be exploited. The first link transmits the CSI from the MSs to the ABS, while the feedback link from the HBS to the ABS sends information on the second-order statistics of the interference signals present at the HBS. For the design of the feedback links different algorithms are considered, featuring both differential [6] and non-differential quantization [7]. The differential algorithm [6] is based on quantization over the space of Hermitian positive definite matrices using geodesic routes, while the nondifferential approach [7] is based on uniform quantization of each coefficient of the matrix to be fed back. The feedback links are assumed delay-free and noise-free, as in [6]-[9]. Since there are two independent feedback links that have to be taken into account for the precoder design at the ABS, the optimum transceiver cannot be computed independently at the 
receivers, and feedback strategies based on quantization of the subspaces spanned by the eigenmodes of the channel matrix, such as those based on codebooks built in the Grassmann manifold [8] are not suitable for this scenario.

This paper is organized as follows. In section II a detailed description of the scenario is provided, including the different channels and the feedback links. Section III presents a review of the differential quantization and feedback algorithm considered in this paper. In section IV the optimum precoder for the ABS is derived fulfilling the interference constraints, and section V presents the simulations results. Finally, section VI concludes the paper.

\section{SySTEM OVERVIEW}

We consider a wireless network with one ABS, one HBS, $K$ MSs and backhauling and access links as depicted in Fig. 1. The ABS has $n_{A}$ transmit antennas, while the HBS has $n_{H}$ and the $i$ th MS has $n_{M_{i}}$ receive antennas, with $\sum_{i=1}^{K} n_{M_{i}}<n_{A}$. The channel matrix from the ABS to the HBS at time instant $t$ is denoted by $\mathbf{H}_{A H}(t) \in \mathbb{C}^{n_{H} \times n_{A}}$, and the channel matrices of the links between ABS and the MSs are represented by $\mathbf{H}_{A M_{i}}(t) \in \mathbb{C}^{n_{M_{i}} \times n_{A}}$. $\mathbf{B} \in \mathbb{C}^{n_{A} \times n_{S}}$ is the precoding matrix of the backhaul link, to be used at the ABS to transmit $n_{S}$ streams to the HBS. $\mathbf{x} \in \mathbb{C}^{n_{S}}$ represents the $n_{S}$ streams of signals to be transmitted from the ABS to the HBS with $\mathbb{E}\left[\mathbf{x x}^{H}\right]=\mathbf{I}$. The additive white Gaussian noise (AWGN) at the HBS is $\mathbf{w} \in \mathbb{C}^{n_{H}}$, with $\mathbb{E}\left[\mathbf{w} \mathbf{w}^{H}\right]=\sigma_{w}^{2} \mathbf{I}$. The received signal at the HBS is, therefore (we drop the dependency with respect to the time index $t$ for the sake of clarity in the notation):

$$
\mathbf{y}=\mathbf{H}_{A H} \mathbf{B} \mathbf{x}+\mathbf{y}_{\text {int }}+\mathbf{w} \in \mathbb{C}^{n_{H}},
$$

where $\mathbf{y}_{\text {int }}$ is the interference received at the HBS with covariance $\mathbf{R}_{\text {int }}$ and can be caused by external sources or, if the MSs is full-duplex, by the signals $\mathbf{x}_{M_{i}}$ transmitted from the MSs. Therefore, the interference plus noise at the HBS, $\mathbf{y}_{\text {int }}+\mathbf{w}$ has a covariance matrix which is expressed as:

$$
\mathbf{R}_{n}=\sigma_{w}^{2} \mathbf{I}+\mathbf{R}_{i n t},
$$

and it is assumed that it can be estimated at the HBS.

\section{A. Channel model}

The scenario described in Fig. 1 contains three different types of propagation channels:

- Channel from the ABS to the HBS: This channel is static, or very slow varying, since both ABS and HBS have no mobility. Therefore the channel response matrix $\mathbf{H}_{A H}$ can be assumed to be known at both ends.

- Channels from the ABS to the MSs: The MSs have a given mobility, which translates into a Doppler-shift. These channels $\mathbf{H}_{A M_{i}}$ are considered to be time varying, and the MSs are assumed to be able to estimate them with the help of pilot symbols.

- Channels from the MSs to the HBS: It is a system design decision that there is no communication between the HBS and the MSs.

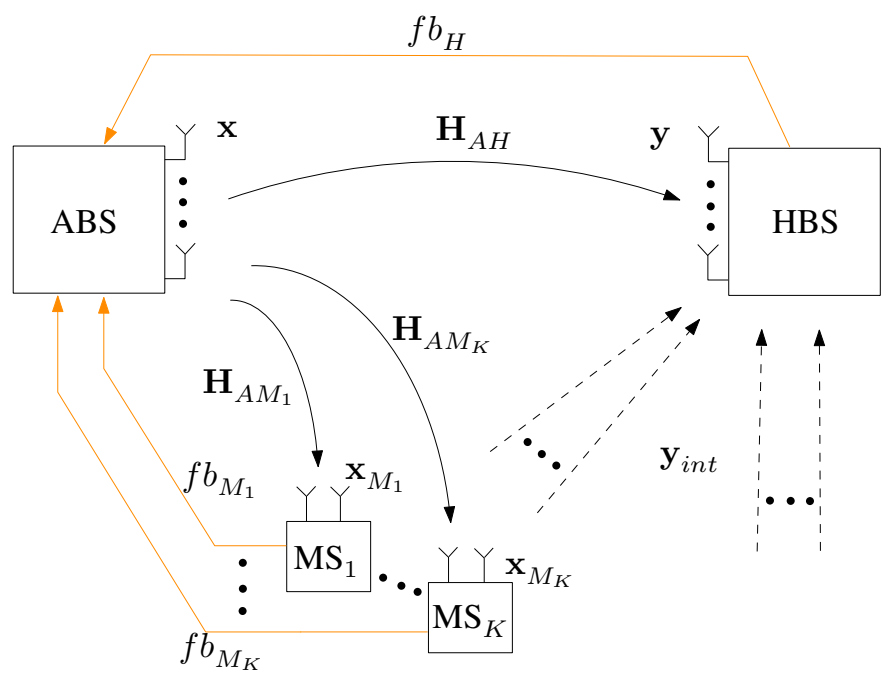

Fig. 1. System model of the wireless backhaul link.

\section{B. Feedback links}

The scenario presented in this paper considers the following feedback links, which are depicted in Fig. 1:

- Link from HBS to ABS $\left(f b_{H}\right)$ : The HBS estimates the interference plus noise correlation matrix $\mathbf{R}_{n}$ and sends it to the ABS through the limited feedback link $f b_{H}$. The ABS then uses this knowledge to minimize the effect of $\mathbf{R}_{n}$ on the performance of the backhaul link by a proper design of the transmit matrix $\mathbf{B}$, as will be explained in section IV of this paper.

- Links from the MSs to the ABS $\left(f b_{M_{i}}\right)$ : The $i$ th MS estimates the propagation channel $\mathbf{H}_{A M_{i}}$ and sends its Gram matrix $\mathbf{H}_{A M_{i}}^{H} \mathbf{H}_{A M_{i}}$ through $f b_{M_{i}}$ to the ABS. This CSI is used at the ABS to reduce the interference caused by the backhaul link over the MSs by a proper design of the precoding matrix $\mathbf{B}$.

\section{Quantization AND FeEdback Algorithm FOR Positive Definite HeRmitian MATRICES}

In [6] we presented a feedback algorithm for single user point-to-point MIMO systems based on the differential quantization of the channel Gram matrix $\mathbf{H}^{H} \mathbf{H}$. It has been proved that, for single user MIMO, the channel Gram matrix contains the sufficient information to design a transmitter that maximizes criteria such as signal to noise ratio (SNR), mutual information, or minimizes bit error rate (BER) or mean square error (MSE) [10]. However, this algorithm can be applied to the general case of quantization and feedback of any positive definite Hermitian matrix, not just the channel Gram matrices. In this paper we will apply the same strategy designed for the channel Gram matrix to the feedback of $\mathbf{R}_{n}$ (feedback link $f b_{H}$ ), which is positive definite and Hermitian by construction 
(see (2)), and also to the feedback of $\mathbf{H}_{A M_{i}}^{H} \mathbf{H}_{A M_{i}}{ }^{1}$ (feedback links $f b_{M_{i}}$ ). This section summarizes this quantization algorithm for positive definite and Hermitian matrices.

\section{A. Preliminaries on some differential geometry definitions}

The objective of the quantization is a Hermitian and positive definite matrix $\mathbf{R}$ of dimensions $m \times m$. As shown in [11] the set of Hermitian positive definite matrices $\mathcal{S}=\left\{\mathbf{R} \in \mathbb{C}^{m \times m}\right.$ : $\left.\mathbf{R}^{H}=\mathbf{R}, \mathbf{R} \succ \mathbf{0}\right\}$ is a convex cone ${ }^{2}$, i.e., $\forall \mathbf{R}_{1}, \mathbf{R}_{2} \in \mathcal{S}, \forall s \geq$ $0, \mathbf{R}_{1}+s \mathbf{R}_{2} \in \mathcal{S}$. This set is described properly by the following definitions [11], [12]:

- Scalar product and norm: The scalar product between two Hermitian matrices $\mathbf{A}$ and $\mathbf{B}$ at any point $\mathbf{R}$ in the set $\mathcal{S}$ is defined as:

$$
\langle\mathbf{A}, \mathbf{B}\rangle_{\mathbf{R}}=\operatorname{Tr}\left(\mathbf{R}^{-1} \mathbf{A} \mathbf{R}^{-1} \mathbf{B}\right)
$$

- Geodesic curve: The geodesic curve $\Gamma(l)$ is the path that connects two points $\mathbf{R}_{1}$ and $\mathbf{R}_{2}$ in $\mathcal{S}$ with minimum distance and with all of its points belonging to $\mathcal{S}$ :

$$
\boldsymbol{\Gamma}(l)=\mathbf{R}_{1}^{1 / 2} \exp (l \mathbf{C}) \mathbf{R}_{1}^{1 / 2} \in \mathcal{S}, \quad \forall l \in \mathbb{R},
$$

where $\mathbf{C}=\mathbf{C}^{H}=\log \left(\mathbf{R}_{1}^{-1 / 2} \mathbf{R}_{2} \mathbf{R}_{1}^{-1 / 2}\right), \boldsymbol{\Gamma}(0)=\mathbf{R}_{1}$, and $\boldsymbol{\Gamma}(1)=\mathbf{R}_{2}$. The direction of the curve at $l=0$ is the derivative at $l=0$, and is given by $\dot{\boldsymbol{\Gamma}}(0)=\mathbf{R}_{1}^{1 / 2} \mathbf{C R}_{1}^{1 / 2}$.

- Geodesic distance: The distance between any two points in $\mathcal{S}$ is given by the length of the geodesic curve that connects them. It is expressed as:

$$
\operatorname{dist}_{g}\left(\mathbf{R}_{1}, \mathbf{R}_{2}\right)=\left(\sum_{i}\left|\log \lambda_{i}\right|^{2}\right)^{1 / 2}=\|\mathbf{C}\|_{F},
$$

where $\left\{\lambda_{i}\right\}$ are the eigenvalues of $\mathbf{R}_{1}^{-1 / 2} \mathbf{R}_{2} \mathbf{R}_{1}^{-1 / 2}$.

In order to understand the generation of curves in the quantization algorithm explained in section III-B, we define the orthogonality between two geodesic curves. Consider two curves $\boldsymbol{\Gamma}_{1}(l)=\mathbf{R}^{1 / 2} \exp \left(l \mathbf{C}_{1}\right) \mathbf{R}^{1 / 2}$ and $\boldsymbol{\Gamma}_{2}(l)=$ $\mathbf{R}^{1 / 2} \exp \left(l \mathbf{C}_{2}\right) \mathbf{R}^{1 / 2}$ that pass through a common point $\mathbf{R}$ at $l=0$. We say that they are orthogonal if their directions at $l=0$ are orthogonal, i.e. $\left\langle\dot{\boldsymbol{\Gamma}}_{1}(0), \dot{\boldsymbol{\Gamma}}_{2}(0)\right\rangle_{\mathbf{R}}=0$, which, from the equation of the derivative and (3), can be written as:

$$
\begin{aligned}
\operatorname{Tr}\left(\mathbf{R}^{-1} \mathbf{R}^{1 / 2} \mathbf{C}_{1} \mathbf{R}^{1 / 2} \mathbf{R}^{-1} \mathbf{R}^{1 / 2} \mathbf{C}_{2} \mathbf{R}^{1 / 2}\right)=0 & \Longleftrightarrow \\
\operatorname{Tr}\left(\mathbf{C}_{1} \mathbf{C}_{2}\right) & =0 .
\end{aligned}
$$

\section{B. Algorithm description}

This quantization algorithm is differential and, as such, the result at any instant $n$ depends on the result of the previous quantization (instant $n-1$ ). The differential nature of the procedure allows to exploit the temporal correlation of the matrix to be quantized and to adapt to dynamic scenarios like

\footnotetext{
${ }^{1}$ Although matrix $\mathbf{H}_{A M_{i}}^{H} \mathbf{H}_{A M_{i}}$ is not strictly positive definite, it is possible to work straightforwardly with extended Gram matrices defined as $\mathbf{H}_{A M_{i}}^{H} \mathbf{H}_{A M_{i}}+\epsilon \mathbf{I}$, for any $\epsilon>0$, which are positive definite by construction. This is done by adding $\epsilon \mathbf{I}$ before the quantization is carried out at the MSs, and subtracting $\epsilon \mathbf{I}$ from the received feedback at the ABS.

${ }^{2}$ Actually, reference [11] is devoted to the case of real matrices, although the results and conclusions can be extended directly to the complex case.
}

TABLE I

MATRIX GEODESIC QUANTIZATION AND FEEDBACK

Initialization: the algorithm starts from the cone vertex: $\widehat{\mathbf{R}}(0)=\mathbf{I}$.

1) The receiver and the transmitter generate a common set of $Q$ random Hermitian matrices $\left\{\tilde{\mathbf{C}}_{i}\right\}_{i=1}^{Q}$ that satisfy the following constraint: $\operatorname{Tr}\left(\tilde{\mathbf{C}}_{m} \tilde{\mathbf{C}}_{j}\right)=\delta_{m j}$, as in (6). Then, each matrix $\tilde{\mathbf{C}}_{i}$ is re-scaled individually by $\Delta$, the quantization step: $\mathbf{C}_{i}=\Delta \tilde{\mathbf{C}}_{i}$.

2) The receiver and the transmitter use $\left\{\mathbf{C}_{i}\right\}_{i=1}^{Q}$ to generate a set of $Q$ geodesic curves $\left\{\boldsymbol{\Gamma}_{i}(l)\right\}_{i=1}^{Q}$ having all of them the same initial point $\widehat{\mathbf{R}}(n-1)$ and with orthogonal directions: $\boldsymbol{\Gamma}_{i}(l)=\widehat{\mathbf{R}}^{1 / 2}(n-1) \exp \left(l \mathbf{C}_{i}\right) \widehat{\mathbf{R}}^{1 / 2}(n-1)$.

The maximum number of orthogonal routes is given by the dimension of the set of Hermitian matrices, i.e., $Q \leq m^{2}$.

3) Each of these geodesic curves is used to generate two candidates for the feedback in the next iteration $\widehat{\mathbf{R}}(n)$, all of them equidistant to $\widehat{\mathbf{R}}(n-1)$. $\left\{\begin{aligned} \widehat{\mathbf{R}}^{(2 i-1)}(n) & =\boldsymbol{\Gamma}_{i}(-1), & & 1 \leq i \leq Q, \\ \widehat{\mathbf{R}}^{(2 i)}(n) & =\boldsymbol{\Gamma}_{i}(1), & & 1 \leq i \leq Q\end{aligned}\right.$

4) Each candidate is evaluated, and the one with the smallest geodesic distance to the actual $\mathbf{R}$ is selected. Its index $i_{F B}$ is sent to the transmitter through the feedback link.

5) The selected matrix will be used for the transmitter design and as the starting point in the next iteration:

$$
\begin{aligned}
i_{F B} & =\arg \min _{i} \operatorname{dist}_{g}\left(\widehat{\mathbf{R}}^{(i)}(n), \mathbf{R}(n)\right), 1 \leq i \leq 2 Q, \\
\widehat{\mathbf{R}}(n) & =\widehat{\mathbf{R}}^{\left(i_{F B}\right)}(n) .
\end{aligned}
$$

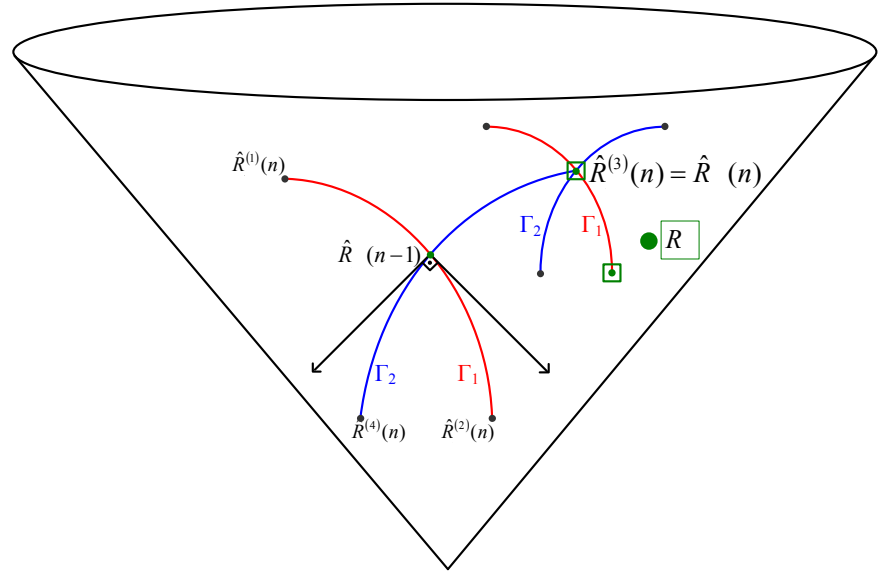

Fig. 2. Differential quantization using 2 bits in the space of positive definite Hermitian matrices.

the one considered in this paper. The mobility of the MSs is taken into account in the algorithm through the quantization step $\Delta$, which can be optimized according to the mobility of the scenario. The objective of the quantization is to minimize the geodesic distance between the actual matrix $\mathbf{R}$ and its fed back estimate $\widehat{\mathbf{R}}$, i.e., its quantized version. The five steps of the feedback algorithm are described in Table I.

Fig. 2 shows the differential quantization process using 2 bits. Starting from $\widehat{\mathbf{R}}(n-1)$, the algorithm generates 2 orthogonal geodesic routes $\boldsymbol{\Gamma}_{1}(l)$ and $\boldsymbol{\Gamma}_{2}(l)$. The four quantization candidates are: $\widehat{\mathbf{R}}^{(1)}(n)=\boldsymbol{\Gamma}_{1}(-1), \widehat{\mathbf{R}}^{(2)}(n)=\boldsymbol{\Gamma}_{1}(1)$, 
$\widehat{\mathbf{R}}^{(3)}(n)=\boldsymbol{\Gamma}_{2}(-1)$, and $\widehat{\mathbf{R}}^{(4)}(n)=\boldsymbol{\Gamma}_{2}(1)$. At the receiver, each candidate is compared to the actual $\mathbf{R}$ and the one with smallest geodesic distance to $\mathbf{R}$ (in this example candidate 3 ) is selected. That is, its index $i_{F B}=3$ is sent to the transmitter through the feedback channel and $\widehat{\mathbf{R}}(n)=\widehat{\mathbf{R}}^{(3)}(n)$. The quantization at next time instant starts from this point, generates 2 orthogonal routes and 4 quantization candidates, selects the candidate with the smallest geodesic distance to $\mathbf{R}$, and so on.

\section{Precoding Matrix Design}

The optimization of the precoding matrix $\mathbf{B}$ in the backhaul link can be done according to several quality criteria, such as maximization of the mutual information, signal to interference plus noise ratio (SINR), or minimization of the BER. Given the constraint of zero-interference to the MSs and taking into account interferant signals at the HBS, the design of $\mathbf{B}$ according to a general design criterion $f\left(\mathbf{B}, \mathbf{H}_{A H}, \mathbf{R}_{n}\right)$, can be formulated as the following maximization problem:

$$
\begin{array}{cc}
\max _{\mathbf{B}} & f\left(\mathbf{B}, \mathbf{H}_{A H}, \mathbf{R}_{n}\right) \\
\text { s.t. } & \operatorname{Tr}\left(\mathbf{B} \mathbf{B}^{H}\right) \leq P_{T}, \\
& \mathbf{H}_{A M_{i}} \mathbf{B}=\mathbf{0}, \forall i(i=1 \ldots K),
\end{array}
$$

where the effect of $\mathbf{R}_{n}$ is considered in the cost function in (7), the constraint on the total power available for the transmission is expressed in (8), and (9) represents the zero-interference constraints to the MSs links. This last constraint can also be written as:

$$
\widetilde{\mathbf{H}}_{A M} \mathbf{B}=\mathbf{0},
$$

where $\widetilde{\mathbf{H}}_{A M}$ is defined as:

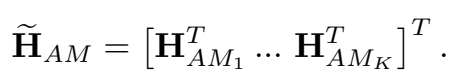

From (10), B is forced to have the following structure:

$$
\mathbf{B}=\mathbf{V}_{0} \widetilde{\mathbf{B}},
$$

where $\widetilde{\mathbf{B}} \in \mathbb{C}^{\left(n_{A}-\sum_{i=1}^{K} n_{M_{i}}\right) \times n_{S}}$ and the orthonormal columns of $\mathbf{V}_{0} \in \mathbb{C}^{n_{A} \times\left(n_{A}-\sum_{i=1}^{K} n_{M_{i}}\right)}$ span the rightnullspace of $\widetilde{\mathbf{H}}_{A M}$ (as in [13]). Since the nullspace of $\widetilde{\mathbf{H}}_{A M}$ is equal to the nullspace of $\widetilde{\mathbf{H}}_{A M}^{H} \widetilde{\mathbf{H}}_{A M}$, and $\widetilde{\mathbf{H}}_{A M}^{H} \widetilde{\mathbf{H}}_{A M}$ can be written as (see (11)):

$$
\widetilde{\mathbf{H}}_{A M}^{H} \widetilde{\mathbf{H}}_{A M}=\sum_{i=1}^{K} \mathbf{H}_{A M_{i}}^{H} \mathbf{H}_{A M_{i}},
$$

matrix $\mathbf{V}_{0}$ can be computed containing as columns the singular vectors associated to the null eigenvalues of $\sum_{i=1}^{K} \mathbf{H}_{A M_{i}}^{H} \mathbf{H}_{A M_{i}}$. Note that from each MS, only the channel Gram matrix $\mathbf{H}_{A M_{i}}^{H} \mathbf{H}_{A M_{i}}$ is needed, and also that the zero forcing applied to each additional MS reduces the degrees of freedom available at the ABS for the communication with the HBS, reducing the performance of the backhaul link.
The total power in (8) can be written as a function of $\widetilde{\mathbf{B}}$ : Since $\mathbf{V}_{0}^{H} \mathbf{V}_{0}=\mathbf{I}$, then $\operatorname{Tr}\left(\mathbf{B B}^{H}\right)=\operatorname{Tr}\left(\widetilde{\mathbf{B}} \widetilde{\mathbf{B}}^{H}\right)$. Taking this into account, the optimization problem can be rewritten as:

$$
\begin{array}{cc}
\max _{\widetilde{\mathbf{B}}} & f\left(\mathbf{V}_{0} \widetilde{\mathbf{B}}, \mathbf{H}_{A H}, \mathbf{R}_{n}\right) \\
\text { s.t. } & \operatorname{Tr}\left(\widetilde{\mathbf{B}} \widetilde{\mathbf{B}}^{H}\right) \leq P_{T} .
\end{array}
$$

This optimization problem can be easily solved now for the different design criteria, such as maximization of the mutual information, SINR, minimization of the BER, etc. We now comment two examples of design criterion $f$, however the same procedure can be applied to other criteria following the same steps [14].

\section{A. Example 1: Maximization of the mutual information:}

In this case the cost function is defined as

$$
\begin{aligned}
f & =\log _{2}\left|\mathbf{I}+\mathbf{B}^{H} \mathbf{H}_{A H}^{H} \mathbf{R}_{n}^{-1} \mathbf{H}_{A H} \mathbf{B}\right|= \\
& =\log _{2}\left|\mathbf{I}+\widetilde{\mathbf{B}}^{H} \mathbf{V}_{0}^{H} \mathbf{H}_{A H}^{H} \mathbf{R}_{n}^{-1} \mathbf{H}_{A H} \mathbf{V}_{0} \widetilde{\mathbf{B}}\right|= \\
& =\log _{2}\left|\mathbf{I}+\widetilde{\mathbf{B}}^{H} \overline{\mathbf{R}}_{H} \widetilde{\mathbf{B}}\right|,
\end{aligned}
$$

where $\overline{\mathbf{R}}_{H}=\mathbf{V}_{0}^{H} \mathbf{H}_{A H}^{H} \mathbf{R}_{n}^{-1} \mathbf{H}_{A H} \mathbf{V}_{0}$.

The solution to the maximization problem (14) for this cost function is known to be [15]:

$$
\widetilde{\mathbf{B}}=\mathbf{V P}^{1 / 2}, \quad \mathbf{P}=\operatorname{diag}\left(p_{1}, \ldots, p_{n_{S}}\right),
$$

where $\mathbf{V}$ consists of $n_{S}$ columns that are the $n_{S}$ eigenvectors of $\overline{\mathbf{R}}_{H}$ associated to its $n_{S}$ maximum eigenvalues $\left\{\lambda_{i}\right\}_{i=1}^{n_{S}}$. The power $\mathbf{P}$ is allocated according to the waterfilling solution $\left(p_{i}=\max \left\{0, \mu-1 / \lambda_{i}\right\}\right.$ where $\mu$ is a constant such that $\left.\sum_{i=1}^{n_{S}} p_{i}=P_{T}\right)$.

B. Example 2: Maximization of the SINR with single beamforming:

In this case the beamforming matrix $\widetilde{\mathbf{B}}$ has only 1 column (therefore the notation $\widetilde{\mathbf{b}}$ will be used), and the cost function is defined as:

$$
f=\left\|\widetilde{\mathbf{b}}^{H} \mathbf{V}_{0}^{H} \mathbf{H}_{A H}^{H} \mathbf{R}_{n}^{-1} \mathbf{H}_{A H} \mathbf{V}_{0} \widetilde{\mathbf{b}}\right\|^{2}=\left\|\widetilde{\mathbf{b}}^{H} \overline{\mathbf{R}}_{H} \widetilde{\mathbf{b}}\right\|^{2},
$$

and the solution to (14) using this criterion as cost function is:

$$
\widetilde{\mathbf{b}}=\sqrt{P_{T}} \mathbf{u}_{\max }\left(\overline{\mathbf{R}}_{H}\right),
$$

where $\mathbf{u}_{\max }(\cdot)$ stands for the unit-norm eigenvector of maximum associated eigenvalue.

\section{Simulation Results}

There are several factors that are considered in this paper and have a direct effect on the performance of the communication in the backhaul link of the proposed scenario. In this section we will first present the performance loss in the link between ABS and HBS when the constraint of zero interference at the MSs is enforced. Then we will analyze the gain obtained when using different feedback techniques to send information of the second order statistics of the 
interference from the HBS to the ABS, as a function of the transmission rate of the feedback link. Finally, the degradation in performance will be simulated for different values of the interfering power and different number of MSs.

For all the simulations a normalized noise power is considered $\left(\sigma_{w}^{2}=1\right)$, and the correlation in time of the propagation channel $\mathbf{H}_{A M}$ is modeled using a first-order autoregressive time variation model described by the following expression:

$$
\mathbf{H}(n)=\rho \mathbf{H}(n-1)+\sqrt{1-\rho^{2}} \mathbf{N}(n),
$$

where $\mathbf{N}(n)$ and the initial value, $\mathbf{H}(n=0)$ are assumed to be independent and composed of i.i.d. zero-mean complex Gaussian entries with unit variance. The time correlation factor $\rho$ models the variability of the channel and depends on the Doppler frequency $f_{D}$ caused by the movement of the MSs through the expression $\rho=J_{0}\left(2 \pi f_{D} \tau\right)$ [16], where $J_{0}$ is the zeroth-order Bessel function of the first kind, $\tau$ corresponds to the time difference between consecutive feedback instants and $f_{r}=\frac{1}{\tau}$ is the feedback rate.

It is important to note that the CSI sent from the MSs to the ABS through the feedback link is quantized and may contain errors, but this does not affect the average performance of the backhaul link ABS-HBS. Imperfect CSI received through the links $f b_{M_{i}}$ degrades the performance of the communication between the ABS and the MSs (which is not the scope of this paper), and only the rank of the transmitted $\mathbf{H}_{A M_{i}}^{H} \mathbf{H}_{A M_{i}}$ reduces the average performance of the backhaul link. For this reason, the accuracy of the feedback links $f b_{M_{i}}$ is not relevant for the simulations and will not be commented explicitly.

First, we consider a full-duplex scenario where $n_{A}=10$, $n_{H}=8$ and there are up to $3 \mathrm{MSs}$ with $n_{M}=2$. Each MS transmits with a power $10 \mathrm{~dB}$ higher than the AWGN. Fig. 3 shows the achievable rate of the ABS-HBS link as a function of the transmit power and averaged over 1000 realizations of the propagation channel, for the cases where the precoding matrix is constrained to put nulls in the directions of the MSs. As expected, the simulation shows that the highest rate is achieved when there are 0 interfering MSs. The slope of the curve is reduced when the number of degrees of freedom of the system decreases. It is interesting to observe that, with this setup, in the presence of 1 interfering MS with 2 antennas, the ABS can do interference nulling at the MS without losing degrees of freedom in the link with the HBS (the curves have the same slope for 0 and $1 \mathrm{MSs}$ ). There is some performance loss due to the fact that system resources are used in the interference nulling but it is a constant loss that does not scale with the transmitted power. The presence of a second MS with 2 antennas does reduce the number of degrees of freedom, and a third MS decreases it further, as shown by the slope of the curves. The maximum degrees of freedom of the ABS-HBS link with interference nulling at all $K$ MSs is $\min \left(n_{A}-\sum_{i=1}^{K} n_{M_{i}}, n_{H}\right)$.

The covariance matrix of the interference plus noise (2) can be estimated at the HBS and sent to the ABS through $f b_{H}$. Fig. 4 shows the gains that can be achieved by the use of

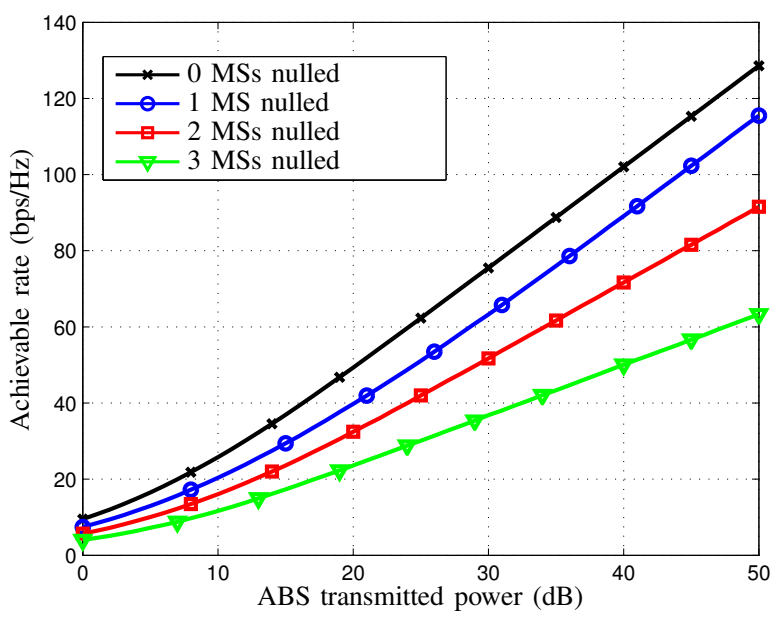

Fig. 3. Effect of interference nulling at the MSs.

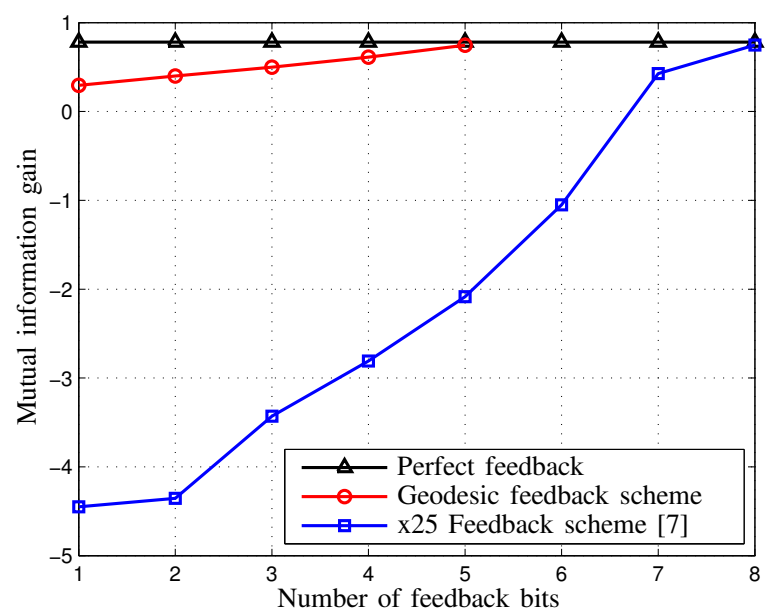

Fig. 4. Mutual information gain vs number of bits of feedback. In the case of [7] the X-axis represents the number of feedback bits for each of the 25 parameters to be quantized.

such feedback link averaged over 3000 channel realizations, as a function of the power of the interfering signal from the MSs and after 30 feedback intervals for a system with $n_{A}=6$, $n_{H}=5$ and 1 interfering MS with $n_{M}=2$. The simulation shows that the differential algorithm outperforms the one based on non-differential quantization [7], which features a uniform quantization of the real and imaginary parts of the elements of $\mathbf{R}_{n}$ (i.e. there are 25 scalar parameters to quantize in this case since $\mathbf{R}_{n}$ has dimensions $5 \times 5$ ), because it is capable of exploiting the correlation in time present in the propagation channel. Also note that having a very inaccurate CSI is worse than having no CSI at all, as shown in the curve corresponding to the non-differential scheme.

The performance in terms of SINR and BER using a BPSK modulation is evaluated as a function of the interfering power in the simulations corresponding to Fig. 5 and Fig. 6, respectively. For these simulations the following setup was 


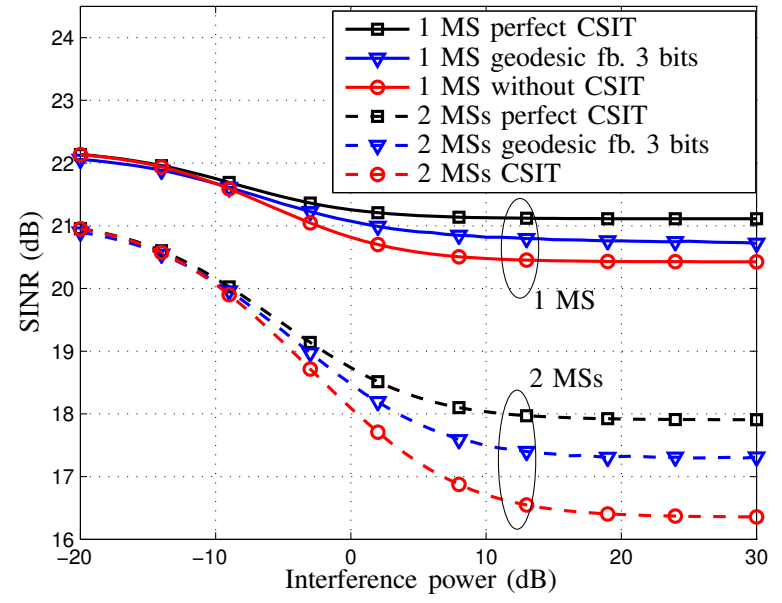

Fig. 5. SINR vs interference power.

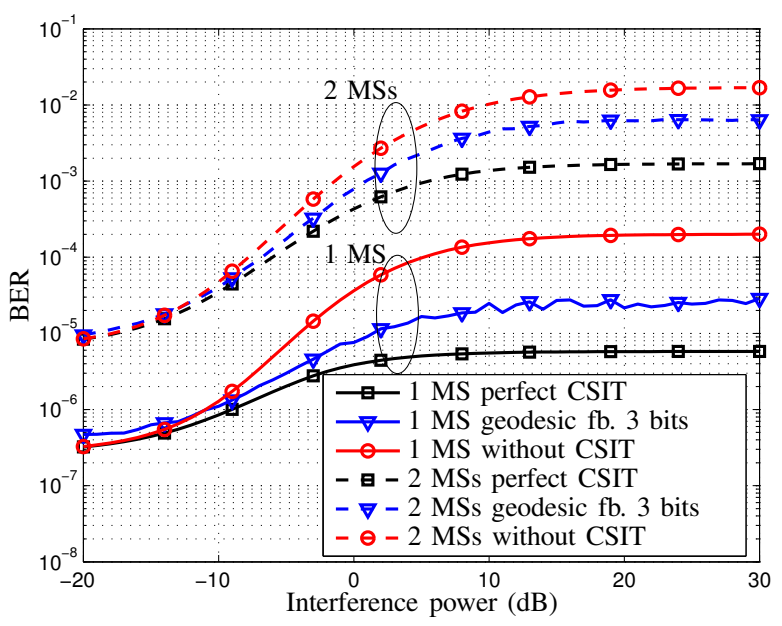

Fig. 6. BER vs interference power.

used: $n_{A}=8, n_{H}=6, n_{M}=2$, and the ABS transmits at a power $10 \mathrm{~dB}$ higher than the AWGN. The curves show how the performance of the backhaul link is degraded by increasing the power of the interfering MSs, and part of this loss can be compensated by using a feedback link with limited capacity to convey the second order statistics of the interfering signal from the HBS to the ABS. The feedback strategy considered is the one described in section III-B, and the results are plotted after 30 feedback intervals and averaged over 6000 channel realizations. These simulations also show that the performance loss due to having to null additional interfering MSs is higher than the loss due to an increase in interfering power of each MS.

\section{CONCLUSIONS}

In this paper we have presented a novel scenario for the backhaul link in an all-wireless network. The communication between a static ABS and a static HBS is considered, in the presence of external interference at the HBS. The scenario features two types of limited feedback links, one from the HBS to the ABS which is used to transmit information on the second order statistics of the interference plus noise at the HBS and another feedback link from each MS to the ABS which sends information of the Grammian of their current channel propagation matrix.

It is a design decision that the link between ABS and HBS does not interfere with the access network and also that the effect of the interference at the HBS is taken into account for the design of the precoding matrix at the ABS. The solution presented in this paper is the optimum precoding matrix given these considerations.

Simulations show the performance of the proposed solution, and the gain achieved by using both a differential and a non-differential quantization algorithm in the feedback links. The differential algorithm achieves better performance because it exploits the geometry of the domain space and also the correlation in time of the propagation channel.

\section{REFERENCES}

[1] I. E. Telatar, "Capacity of multi-antenna Gaussian channels," European Trans. on Telecommunications, vol. 10, no. 6, pp. 585-595, Nov. 1999.

[2] S. M. Alamouti, "A simple transmit diversity technique for wireless communications," IEEE Journal on Selected Areas in Communications, vol. 16, no. 8, pp. 1451-1458, Oct. 1998.

[3] A. Goldsmith, S. Jafar, N. Jindal, and S. Vishwanath, "Capacity limits of MIMO channels," IEEE Journal on Selected Areas in Communications, vol. 21, no. 5, pp. 684-702, Jun. 2003.

[4] IEEE 802.16-2004 Standard for Local and Metropolitan Area Networks Part 16: Air Interface for Fixed Broadband Wireless Access Systems, IEEE 802.16 Std., Oct. 2004.

[5] J. G. Andrews, A. Ghosh, and R. Muhamed, Fundamentals of WiMAX: Understanding Broadband Wireless Networking. Prentice Hall, 2007.

[6] D. Sacristán-Murga and A. Pascual-Iserte, "Differential feedback of MIMO channel correlation matrices based on geodesic curves," in Proc. IEEE ICASSP'09, Apr. 2009.

[7] C.-B. Chae, D. Mazzarese, N. Jindal, and R. W. Heath, "Coordinated beamforming with limited feedback in the MIMO broadcast channel," IEEE Journal on Selected Areas in Communications, vol. 26, no. 8, pp. 1505-1515, Oct. 2008.

[8] D. J. Love, R. W. Heath, and T. Strohmer, "Grassmannian beamforming for multiple-input multiple-output wireless systems," IEEE Trans. on Information Theory, vol. 49, no. 10, pp. 2735-2747, Oct. 2003.

[9] R. W. Heath, T. Wu, and A. C. K. Soong, "Progressive refinement of beamforming vectors for high-resolution limited feedback," EURASIP Journal on Advances in Signal Processing, pp. 1-13, 2009.

[10] M. Payaró and D. P. Palomar, "On optimal precoding in linear vector Gaussian channels with arbitrary input distribution," in Proc. IEEE International Symposium on Information Theory (ISIT'09), Jul. 2009.

[11] M. Talih, "Geodesic Markov chains on covariance matrices," Statistical and Applied Mathematical Sciences Institute, Tech. Rep., Mar. 2007.

[12] X. Pennec, P. Fillard, and N. Ayache, "A Riemannian framework for tensor computing," International Journal of Computer Vision, vol. 66, no. 1, pp. 41-66, Jan. 2006, a preliminary version appeared as INRIA Research Report 5255, July 2004.

[13] Q. H. Spencer, A. L. Swindlehurst, and M. Haardt, "Zero-forcing methods for downlink spatial multiplexing in multiuser MIMO channels," IEEE Trans. on Signal Processing, vol. 52, no. 2, pp. 461-471, Feb. 2004.

[14] D. P. Palomar, J. M. Cioffi, and M. A. Lagunas, "Joint Tx-Rx beamforming design for multicarrier MIMO channels: a unified framework for convex optimization," IEEE Trans. on Signal Processing, vol. 51, no. 9 , pp. 2381-2401, Sep. 2003.

[15] T. M. Cover and J. A. Thomas, Elements of Information Theory. WileyInterscience, 2006.

[16] R. Steele and L. Hanzo, Eds., Mobile Radio Communications, 2nd ed. John Wiley \& Sons, 1999. 